\title{
Retrobulbar blood flow and visual field alterations after acute ethanol ingestion
}

This article was published in the following Dove Press journal:

Clinical Ophthalmology

16 August 2013

Number of times this article has been viewed

\author{
Anke Weber \\ Andreas Remky \\ Marion Bienert \\ Klaudia Huber-van der \\ Velden \\ Thomas Kirschkamp \\ Corinna Rennings \\ Gernot Roessler \\ Niklas Plange \\ Department of Ophthalmology, \\ RWTH Aachen University, Aachen, \\ Germany
}

Background: The purpose of this study was to test the effect of ethyl alcohol on the koniocellular and magnocellular pathway of visual function and to investigate the relationship between such visual field changes and retrobulbar blood flow in healthy subjects.

Methods: In 12 healthy subjects (mean age $32 \pm 4$ years), color Doppler imaging, shortwavelength automated perimetry, and frequency doubling perimetry was performed before and 60 minutes after oral intake of $80 \mathrm{~mL}$ of $40 \mathrm{vol} \%$ ethanol. Mean and pattern standard deviations for short-wavelength automated and frequency doubling perimetry were assessed. End diastolic velocity (EDV) and peak systolic velocity (PSV) were measured in the central retinal and ophthalmic arteries using color Doppler imaging. Systemic blood pressure, heart rate, intraocular pressure, and blood alcohol concentration were determined.

Results: Mean PSV and EDV in the central retinal artery showed a significant increase after alcohol intake ( $P=0.03$ and $P=0.02$, respectively). Similarly, we found a significant acceleration of blood flow velocity in the ophthalmic artery $(P=0.02$ for PSV; $P=0.04$ for EDV). Mean intraocular pressure decreased by $1.0 \mathrm{mmHg}$ after alcohol ingestion $(P=0.01)$. Retinal sensitivity in short-wavelength automated perimetry did not alter, whereas in frequency doubling perimetry, the mean deviation decreased significantly. Systolic and diastolic blood pressure did not change significantly. Mean blood alcohol concentration was $0.38 \pm 0.16 \mathrm{~g} / \mathrm{L}$.

Conclusion: Although ethanol is known to cause peripheral vasodilation, our subjects had no significant drop in systemic blood pressure. However, a significant increase of blood flow velocity was seen in the retrobulbar vessels. Regarding visual function, moderate alcohol consumption led to reduced performance in the magnocellular visual system tested by frequency doubling perimetry, but had no effect on short-wavelength automated perimetry.

Keywords: visual field, retrobulbar blood flow, frequency doubling perimetry, short-wavelength automated perimetry

\section{Introduction}

Ethanol is a commonly used psychoactive substance. The systemic and central effects of ethanol have been investigated thoroughly. Several studies have shown that moderate and substantial doses of alcohol influence several visual and oculomotor functions. Impairments have been shown when convergence, accommodation, stereoacuity, visual acuity, visual fields, color vision, and contrast sensitivity function were tested. ${ }^{1-10}$

Moderate but regular consumption of alcohol is part of the normal life style in many industrial nations and might have an impact on clinical routine examinations, including those performed by ophthalmologists. ${ }^{11,12}$ The purpose of this study was on the one hand to investigate further the influence of alcohol consumption on the visual system using short-wavelength automated perimetry and frequency doubling technology, and
Correspondence: Niklas Plange Department of Ophthalmology, RWTH Aachen University, Pauwelsstrasse 30, Aachen 52074, Germany

Tel +49 24I 808 8191

Fax +49 24I 8082408

Email nplange@ukaachen.de 
on the other to determine whether alcohol used in relatively low doses leads to alteration in retrobulbar blood flow. Both frequency doubling and short-wavelength automated perimetry have been established more recently and have been shown to be useful for indicating visual field defects associated with various diseases earlier than standard whiteon-white perimetry. ${ }^{13-20}$ Therefore, we hypothesized that alteration due to mild alcohol consumption as commonly practiced in Europe, if present at all, might show up in these tests, which are known to detect subtle visual disturbances. In addition, distinct changes of frequency doubling and short-wavelength automated perimetry due to ethanol ingestion might be relevant concerning the interpretation of early visual field damage in glaucomatous optic neuropathy. Additional retrobulbar blood flow measurements were performed noninvasively using color Doppler imaging to investigate whether detectable changes in visual performance are associated with alterations in ocular perfusion or whether they are unrelated.

\section{Materials and methods}

All subjects included in this prospective study were medical doctors and staff of the ophthalmology department at the University Hospital in Aachen and participated in the development of the study design. Twelve healthy subjects (seven women and five men, of mean age $32 \pm 4$ years) with no history of alcoholism or alcohol-related problems were included in this study. No systemic or local medication (except contraceptives) was being used by the subjects. Medical history did not reveal systemic cardiovascular disease or risk factors (ie, arterial hypertension, diabetes mellitus, disorders of fat metabolism, any cerebrovascular or cardiovascular events). Subjects known to be regular nicotine abusers $(n=3)$ did not consume nicotine for at least 24 hours prior to the study.

All participants were aware of the risks of mild alcohol consumption and familiar with the tests that were performed. The research followed the tenets of the Declaration of Helsinki. The experiment was performed during a single afternoon, before which a light lunch without alcohol consumption was allowed. All 12 subjects had best corrected visual acuity of 1.0 (20/20). A slit-lamp examination, including funduscopy, was performed for all subjects and found to be normal. One eye was randomly selected for the study. All subjects were familiar with and had performed short-wavelength automated perimetry and frequency doubling perimetry before.

Each experiment began with baseline measurements, including visual field testing with frequency doubling perimetry and short-wavelength automated perimetry, color Doppler imaging, and measurements of systemic blood pressure, heart rate, and intraocular pressure (Goldmann applanation tonometry). Alcohol ( $80 \mathrm{~mL}$ of $40 \mathrm{vol} \%$ ethanol [Schnaps, Schladerer Williams Pear]) was consumed within a 10-minute period afterwards. Forty-five to 60 minutes after ingestion of alcohol, $3 \mathrm{~mL}$ blood samples were taken from the cubital vein followed immediately by a repetition of the battery of tests, performed in the same order. Blood alcohol levels were determined by gas chromatography and expressed in g/L. All ophthalmic examinations including color Doppler imaging were performed by one experienced ophthalmologist (AW).

\section{Short-wavelength automated perimetry}

The principle of short-wavelength automated perimetry is the isolation of the short-wavelength-sensitive cones by suppression of the other cone types using a yellow background. Short-wavelength automated perimetry was performed using a commercially available Humphrey field analyzer (Zeiss Humphrey Systems, Dublin, CA, USA). Short-wavelength automated perimetry utilizes a Goldmann size V (1.8 degree visual angle) narrow-band blue stimulus with a peak transmission of $440 \mathrm{~nm}$ on a yellow background illumination $\left(100 \mathrm{~cd} / \mathrm{m}^{2}\right)$. The duration of the stimulus amounts to $200 \mathrm{msec}$. In this study, the 24-2 program was applied with the full threshold strategy. Tests with false positive or false negative responses exceeding $20 \%$ of all answers were excluded. Global indices (mean deviation and pattern standard deviation) and test duration were used for statistical analysis.

\section{Frequency doubling technology}

Frequency doubling perimetry measures contrast sensitivity using a perceptual effect encountered when coarse vertical grating targets are counterphase-flickered at a rapid rate (frequency doubling illusion). ${ }^{13}$ We performed frequency doubling perimetry using Humphrey-Zeiss frequency doubling technology (Carl Zeiss Meditec, Dublin, CA, USA; Welch Allyn, Skaneateles Falls, NY, USA) with a fullthreshold C-20 program in all subjects before and after alcohol ingestion. The test uses a square target which is 10 degrees wide and consists of alternating dark and light stripes (spatial frequency 0.25 cycles per degree). This grating is counterphase-flickered at $25 \mathrm{~Hz}$, and the contrast of the stripes is changed to determine the subject's contrast threshold. In the C-20 program, 17 locations in the 20 degree field are tested using 16 approximately 10 degree square targets, along with an additional circular target centered over the macula. 


\section{Color Doppler imaging}

Color Doppler imaging combines a simultaneous B-mode ultrasound image with colored represented movements based on Doppler frequency shifts and enables assessment of blood flow velocities (peak systolic velocity and end diastolic velocity) in the retrobulbar vessels. A color Doppler imaging system (Siemens Sonoline, Washington, DC, USA) with a $7.5 \mathrm{mHz}$ transducer was used to measure retrobulbar blood flow velocity. Ultrasonographic evaluation was performed by an experienced ultrasonographer (AW) who was masked to the subject's mean sensitivity in shortwavelength automated perimetry and frequency doubling technology. Peak systolic velocity (PSV) and end diastolic velocity (EDV) were measured from the Doppler signal in the central retinal and ophthalmic arteries. In addition to the velocities, the Pourcelot resistive index was calculated for all vessels as resistive index $=(\mathrm{PSV}-\mathrm{EDV}) / \mathrm{PSV}$. The resistive index is a measure of peripheral vascular resistance and is less dependent than measures of absolute velocities on the Doppler angle, machine setting, and physiologic conditions, such as hypotension and hypertension. ${ }^{21,22}$

During measurements, the subject was in the supine position and the ultrasound transducer coupled with sterile ophthalmic gel was applied to the upper lids with minimal pressure to avoid artifacts. Evaluation of the vessels always began with an image of the optic nerve, which is the most useful landmark for identifying retrobulbar vessels. The central retinal artery can be found in the retrolaminar region of the optic nerve (approximately 10-20 $\mathrm{mm}$ behind the globe). The ophthalmic artery is usually found nasally to the optic nerve, approximately $25 \mathrm{~mm}$ behind the globe.

\section{Statistical analysis}

Statistical analysis was performed using the StatView ${ }^{\mathrm{TM}}$ (SAS Institute Inc, Cary, NC, USA). Paired $t$-tests were used to assess the significance of differences for blood flow measurements and mean deviation and patterned standard deviation for short-wavelength automated perimetry and frequency doubling perimetry before and after alcohol ingestion. $P$ values $<0.05$ were considered to be statistically significant. Correlations were tested using the Fisher $\mathrm{r}$ to $\mathrm{z}$ test.

\section{Results}

The mean blood alcohol level was $0.38 \pm 0.16 \mathrm{~g} / \mathrm{L}$ 45-60 minutes after alcohol ingestion. Systolic and diastolic blood pressure did not show a significant alteration after alcohol ingestion (systolic blood pressure before alcohol was $131 \pm 14 \mathrm{mmHg}$ and after alcohol was $127 \pm 9 \mathrm{mmHg}$;
$P=0.19$; diastolic BP before alcohol was $74 \pm 12 \mathrm{mmHg}$ and after alcohol was $72 \pm 8 \mathrm{mmHg} ; P=0.67)$. Similarly, no significant change in heart rate was seen (before ethanol $70 \pm 12$ beats/minute; after ethanol $68 \pm 9$ beats/minute; $P=0.59$ ). However, intraocular pressure was significantly decreased by $1.0 \mathrm{mmHg}$, from $13 \pm 2 \mathrm{mmHg}$ to $12 \pm 2 \mathrm{mmHg}$ $(P=0.015)$.

\section{Frequency doubling technology perimetry}

Mean deviation, pattern standard deviation, and test durations before and after alcohol ingestion are shown in Table 1. There was a significant reduction in mean deviation after alcohol ingestion (Table 1). No significant alteration was found for the pattern standard deviation after alcohol ingestion $(P=0.7)$. Duration of testing times showed a trend towards longer testing times after alcohol administration, but these did not reach statistical significance (Table 1).

\section{Short-wavelength automated perimetry}

Mean deviation in short-wavelength automated perimetry was not significantly altered after alcohol ingestion (before alcohol $-0.44 \pm 2.0 \mathrm{~dB}$, after alcohol $-0.42 \pm 2.0 \mathrm{~dB} ; P=0.94$ ) Analogously, pattern standard deviation did not demonstrate a significant change after alcohol ingestion (before alcohol $2.45 \pm 1.0 \mathrm{~dB}$, after alcohol $2.53 \pm 0.75 ; P=0.62$ ). Mean test duration was prolonged, but did not reach statistical significance (before alcohol $9.9 \pm 1.1$ minutes, after alcohol $10.1 \pm 1.3$ minutes; pattern standard deviation $=0.44$ ).

\section{Color Doppler imaging}

PSV, EDV, and resistive index in the central retinal and ophthalmic arteries are shown in Table 2. Ethanol intake led to a significant increase in PSV and EDV and to a significant decrease in the resistive index in both vessels (Table 2). When correlation calculations were performed for the changes in functional and circulatory parameters, we found that changes in end diastolic velocity in the central retinal artery were correlated with changes in mean deviation in short-wavelength automated perimetry $(\mathrm{r}=-0.62$;

Table I Mean deviation, pattern standard deviation, and test duration (mean \pm standard deviation) in frequency doubling perimetry before and after alcohol ingestion

\begin{tabular}{llcl}
\hline & Before alcohol & After alcohol & P value \\
\hline MD (dB) & $0.66 \pm 1.5$ & $-0.49 \pm 1.9$ & 0.007 \\
PSD (dB) & $3.74 \pm 0.48$ & $3.86 \pm 0.97$ & 0.7 \\
Test duration (minutes) & $4.23 \pm 0.15$ & $4.38 \pm 0.25$ & 0.06
\end{tabular}

Abbreviations: MD, mean deviation; PSD, pattern standard deviation. 
Table 2 Mean PSV, EDV, and resistive index (mean \pm standard deviation) in the central retinal and ophthalmic arteries before and after alcohol ingestion

\begin{tabular}{lcll}
\hline & Before alcohol & After alcohol & $P$ value \\
\hline PSV in OA $(\mathrm{cm} / \mathrm{sec})$ & $36.8 \pm 6.7$ & $41.4 \pm 8.0$ & 0.017 \\
EDV in OA $(\mathrm{cm} / \mathrm{sec})$ & $8.7 \pm 1.9$ & $11.6 \pm 4.0$ & 0.039 \\
RI in OA & $0.76 \pm 0.04$ & $0.72 \pm 0.07$ & 0.04 \\
PSV in CRA $(\mathrm{cm} / \mathrm{sec})$ & $10.8 \pm 1.5$ & $11.6 \pm 1.1$ & 0.032 \\
EDV in CRA $(\mathrm{cm} / \mathrm{sec})$ & $3.9 \pm 0.7$ & $4.4 \pm 0.7$ & 0.022 \\
RI in CRA & $0.63 \pm 0.05$ & $0.62 \pm 0.05$ & 0.015 \\
\hline
\end{tabular}

Abbreviations: CRA, central retinal artery; OA, ophthalmic artery; RI, resistive index; EDV, end diastolic velocity; PSV, peak systolic velocity.

$P=0.029$ ). All other correlation calculations of the changes lacked statistical significance.

\section{Discussion}

The effect of alcohol ingestion on visual function and ocular hemodynamics has been investigated previously. ${ }^{1-10}$ In our study, visual fields were evaluated using frequency doubling perimetry and short wavelength automated perimetry, known to be more susceptible to subtle changes in visual performance. ${ }^{15,19}$

Our hypothesis was that moderate consumption of alcohol would lead to reduction in visual function. Indeed, our results show a significant decrease for mean deviation in frequency doubling perimetry after alcohol consumption and a slight increase in pattern standard deviation, indicating that there is mainly a general depression in retinal sensitivity with no particular presence of focal defects. The frequency doubling percept is thought to reflect the response of a subset of magnocellular retinal ganglion cells with nonlinear properties (My cells), although others dispute the evidence for this subset. ${ }^{23,24}$ However, disregarding the existence of this special subset of cells, the magnocellular system itself might still be subject to selective loss and lower redundancy. Our results indicate that the magnocellular visual pathway tested by frequency doubling perimetry might be susceptible even to moderate doses of alcohol. To our minds, these are unique results because the effect of alcohol on the frequency doubling percept has only been investigated once before and the results are in disagreement with ours. ${ }^{25}$ Puell and Barrio ${ }^{25}$ could not detect significant differences in frequency doubling perimetry mean sensitivity during mild alcohol consumption compared with the sober sessions, which were at least two days apart. In our protocol, the tests were repeated during one day, directly before alcohol consumption and 60 minutes afterwards. This might be the reason why we could detect very subtle changes in our study.
We also tested the implications of moderate alcohol consumption on short-wavelength cone sensitivity using short-wavelength automated perimetry. We assumed that alcohol consumption would in particular lead to alterations in blue-sensitive cones because they have been shown to be more susceptible to toxic substances, including ethanol. ${ }^{1,2,26-28}$ Moreover, it has been suggested that visual field defects associated with glaucoma present years earlier in shortwavelength automated perimetry than in standard automated perimetry. ${ }^{15}$ However, our results failed to detect decreased retinal sensitivity in short-wavelength automated perimetry after moderate alcohol consumption.

In previous studies, in which blue-sensitive cones were found transiently affected after alcohol consumption, blood alcohol concentrations were higher than in our study (about $0.7 \mathrm{~g} / \mathrm{L}$ compared with $0.38 \mathrm{~g} / \mathrm{L}$ in our study) ${ }^{26,27}$ Secondly, the effect of alcohol on blue-sensitive cones was tested using different methods compared with our approach to testing visual field performance (eg, Farnsworth-Munsell 100-Hue test or the Panel D-15 test). Finally, the missing impact on short-wavelength automated perimetry might be explained by the higher intraindividual variability shown for short-wavelength automated perimetry, which might mask significant changes. ${ }^{29,30}$

Our data suggest that the effect of alcohol on distinct visual pathways might be different. The magnocellular visual pathway seems to be more susceptible to moderate doses of alcohol, whereas the koniocellular system might be more robust. Whether this difference in susceptibility concerning the magnocellular and koniocellular pathway shows a dosedependence needs to be investigated in future studies.

In addition to visual performance, we investigated retrobulbar blood flow after alcohol ingestion using color Doppler imaging, a noninvasive method to measure blood flow velocity. Several studies have investigated ocular blood flow after alcohol ingestion using different methods and with inconsistent outcomes. ${ }^{10,31,32}$ Our results show a significant increase in PSV and EDV in the central retinal and ophthalmic arteries, and additionally a decrease in resistive indices in both vessels. As with previously published data, this indicates that intake of moderate doses of alcohol is associated with distal vasorelaxation in the central retinal and ophthalmic arteries, despite the autoregulatory mechanism that is known for the retinal circulation. ${ }^{33}$ Similarly, improvement of blood flow in the optic nerve head was found after consumption of one bottle of beer. ${ }^{32}$ Conflicting results were reported by Harris et al who found that acute ethanol consumption did not change retrobulbar blood flow velocities or resistive indices, although 
they used higher breath alcohol levels. ${ }^{10}$ These discrepancies might be explained by dose-dependency of the vascular effects of ethanol. Mathew and Wilson concluded after a thorough overview of the previous literature that most human studies suggest occurrence of cerebral vasodilation with small doses of alcohol and cerebral vasoconstriction with higher doses. ${ }^{34}$ This conclusion might also be true for ocular blood flow.

Another purpose of this study was to investigate whether low doses of ethanol would lead to a decrease in retinal function independent of alterations in ocular perfusion. When we correlated alterations in blood flow velocity with changes in mean deviation and pattern standard deviation in frequency doubling perimetry and short-wavelength automated perimetry, we only found a negative correlation for mean deviation in frequency doubling perimetry and EDV in the central retinal artery, indicating that, with better perfusion, visual performance in frequency doubling perimetry is reduced. Thus, we could not find a general association between retrobulbar blood flow alterations and changes in visual field perception. This finding might indicate that the decrease in mean deviation shown in frequency doubling perimetry was either due to a direct or indirect influence on the inner retinal layers, or to general central depressant effects that overrule the potential beneficial hemodynamic ones. It is well known that alcohol affects the GABA, dopamine, and opioid systems, although it is difficult to attribute changes found in the visual system to any specific neurotransmitter or modulator. ${ }^{8,35}$ The attenuating effect on the GABA system could partly account for the depression in visual function in frequency doubling perimetry, but does not explain the missing decrease in visual performance in short-wavelength automated perimetry. To our surprise, no changes were detected in short-wavelength automated perimetry and only subtle changes in frequency doubling perimetry after mild alcohol ingestion. Therefore, a larger study sample with varying blood alcohol doses might be beneficial to detect a possible association of retrobulbar blood flow and frequency doubling perimetry or short-wavelength automated perimetry. In addition, such an association might be different in patients with glaucomatous visual field defects.

To summarize, in this experiment we found a significant decrease in general sensitivity for frequency doubling perimetry, but no alterations for short-wavelength automated perimetry. These functional changes were accompanied by an increase in blood flow velocity, but lacked a direct correlation with each other. Therefore, we postulate that functional disturbances are mostly due to the pharmacologic effects of alcohol which cannot be equaled by enhanced local perfusion.
Further investigation in greater numbers of subjects is needed to explore the effect of alcohol in different and higher doses on these sensitive diagnostic tools. Finally, it is important that clinical ophthalmologists and general practitioners know that alcohol consumption might alter the results of routine examination using standard clinical tests, such as frequency doubling perimetry.

\section{Disclosure}

The authors report no conflicts of interest in this work.

\section{References}

1. Remky H. Augenärztliche Befunde bei akuter Alkoholeinwirkung [Ophthalmologic findings after alcohol consumption]. In: Laves W, Bitzel F, editors. Der Straßenverkehrsunfall [The road traffic accident]. Stuttgart, Germany: Ferdinand Enke Verlag; 1956. German.

2. Gramberg-Danielsen B. Farbenuntüchtigkeit und Straßenverkehr [Unfitness to drive and traffic]. Z Verk Med. 1965;11:1-12. German.

3. Riedel KG, Gilg TH, Liebhardt E. Wahrnehmungsstörungen im peripheren Gesichtsfeld unter Alkoholeinfluß [Perceptual disorders of the peripheral visual field caused by alcohol. Studies with the automatic Octopus perimeter]. Klin Monatsbl Augenheilkd. 1985; 186:279-283.

4. Zrenner E, Riedel KG, Adamczyk R, Gilg T, Liebhardt E. Effects of alcohol on the electrooculogram and color vision. Doc Ophthalmol. 1986;63:305-312.

5. Zulauf M, Flammer J, Signer C. Short-term influence of alcohol on spatial brightness contrast sensitivity. Ophthalmologica. 1988;197:159-165.

6. Wild JM, Betts TA, Shaw DE. The influence of a social dose of alcohol on the central visual field. Jpn J Ophthalmol. 1990;34:291-297.

7. Hill JC, Toffolon G. Effects of alcohol on sensory and sensorimotor visual function. J Stud Alcohol. 1990;51:108-113.

8. Watten RG, Lie I. Visual function and acute ingestion of alcohol. Ophthalmic Physiol Opt. 1996;16:460-466.

9. Nicholson ME, Andre JT, Tyrell RA, Wang M, Leibowitz HW. Effects of moderate dose alcohol on visual contrast sensitivity for stationary and moving targets. $J$ Stud Alcohol. 1995;56:261-266.

10. Harris A, Swartz D, Engen D, et al. Ocular hemodynamic effects of acute ethanol ingestion. Ophthalmic Res. 1996;28:193-200.

11. Knüfer H, Kraus L. Epidemiologische und ökonomische Aspekte des Alkoholismus [Epidemiological and economic aspects of alcoholism]. Dtsch Ärztebl. 2002;99:936-945. German. Available from: http://www. aerzteblatt.de/archiv/31093. Accessed May 21, 2013.

12. http://www.bzga.de Bundeszentrale für gesundheitliche Aufklärung [Federal centre for health education]. German. Accessed May 21, 2013.

13. Kelly DH. Frequency doubling in visual responses. J Opt Soc Am. 1966;56:1628-1633.

14. Quigley HA, Sanchez RM, Dunkelberger GR, Hernault NL, Baginski TA. Chronic glaucoma selectively damages large optic nerver fibers. Invest Ophthalmol Vis Sci. 1987;28:913-920.

15. Johnson CA, Adams AJ, Casson EJ, Brandt JD. Blue-on-yellow perimetry can predict the development of glaucomatous visual field loss. Arch Ophthalmol. 1993;111:645-650.

16. Johnson CA. The Glenn A Fry Award Lecture: early losses of visual function in glaucoma. Optom Vis Sci. 1995;72:359-370.

17. Wild JM. Short-wavelength automated perimetry. Acta Ophthalmol Scand. 2001;79:546-559.

18. Girkin CA, McGwin G Jr, DeLeon-Ortega J. Frequency doubling perimetry in non-arteritic ischaemic optic neuropathy with altitudinal defects. Br J Ophthalmol. 2004;88:1274-1279.

19. Medeiros FA, Sample PA, Weinreb RN. Frequency doubling technology perimetry abnormalities as predictors for glaucomatous visual field loss. Am J Ophthalmol. 2004;137:863-871. 
20. Coralla G, Cicinella S, Papadia M, Bandini F, Ucelli A, Calabria G. Conventional perimetry, short-wavelength automated perimetry, frequency doubling perimetry, and visual evoked potentials in the assessment of patients with multiple sclerosis. Eur J Ophthalmol. 2005; 15:730-738.

21. Friedman E, Krupsky S, Lane AM, et al. Ocular blood flow velocity in age-related macular degeneration. Ophthalmology. 1995;102: 640-646.

22. Williamson TH, Harris A. Color Doppler imaging of the eye and orbit. Surv Ophthalmol. 1996;40:225-267.

23. Maddess T, Henry GH. Performance of nonlinear visual units in ocular hypertension and glaucoma. Clin Vis Sci. 1992;7:371-383.

24. White AJ, Sun H, Swanson WH, Lee BB. An examination of physiological mechanism underlying the frequency doubling illusion. Invest Ophthalmol Vis Sci. 2002;43:3590-3599.

25. Puell MC, Barrio A. Effect of driver distraction and low alcohol concentrations on useful field of view and frequency-doubling technology perimetry. Acta Ophthalmol. 2008;86:634-641.

26. Russell RM, Carney EA, Feiock K, Garrett M, Karwoski P. Acute ethanol administration causes transient impairment of blue-yellow color vision. Alcohol Clin Exp Res. 1980;4:396-399.

27. Zrenner E. Electrophysiological characteristics of the blue-sensitive mechanism: test of a model of cone interaction under physiological and pathological conditions. Doc Ophthalmol Proc Ser. 1982;33: 103-125.
28. Greenstein VD, Hood DC, Ritch R, Steinberger D, Carr RE. Blue cone pathway vulnerability in retinitis pigmentosa, diabetes and glaucoma. Invest Ophthalmol Vis Sci. 1989;30:1732-1737.

29. Kwon YH, Park HJ, Jap A, Ugurlu S, Caprioli J. Test-retest variability of blue-on-yellow perimetry is greater than white-on-white perimetry in normal subjects. Am J Ophthalmol. 1998;126:29-36.

30. Remky A, Arend O. Intra-individual variability of blue-yellow perimetry. Ophthalmologe. 2000;97:774-780. German.

31. Dhasmana D, Herber L, Patel V, Chen HC, Jones M, Kohner EM. The effect of acute ethanol consumption on the human retinal circulation: a study in diabetic and non-diabetic subjects. Eur J Ophthalmol. 1994;4: 144-150.

32. Kojima S, Sugiyama T, Koyima M, Azuma I, Ito S. Effect of the consumption of ethanol on the microcirculation of the human optic nerve head in the acute phase. Jpn J Ophthalmol. 2000;44:317-324.

33. Spencer JA, Guissani DA, Moore PJ, Hanson MA. In vitro validation of Doppler indices using blood flow and water. J Ultrasound Med. 1991;10:305-308

34. Mathew RJ, Wilson WH. Substance abuse and cerebral blood flow. Am J Psychiatry. 1991;148:292-305.

35. Ogawa T, Kato H, Ito S. Studies on inhibitory neurotransmission in visual cortex in vitro. In: Pettigew JD, Sanderson KJ, Levick WR, editors. Visual Neuroscience. Cambridge, UK: Cambridge University Press; 1986.
Clinical Ophthalmology

\section{Publish your work in this journal}

Clinical Ophthalmology is an international, peer-reviewed journal covering all subspecialties within ophthalmology. Key topics include: Optometry; Visual science; Pharmacology and drug therapy in eye diseases; Basic Sciences; Primary and Secondary eye care; Patient Safety and Quality of Care Improvements. This journal is indexed on

\section{Dovepress}

PubMed Central and CAS, and is the official journal of The Society of Clinical Ophthalmology (SCO). The manuscript management system is completely online and includes a very quick and fair peer-review system, which is all easy to use. Visit http://www.dovepress.com/ testimonials.php to read real quotes from published authors. 